\title{
Equine pregnancy-specific glycoprotein CEACAM49 secreted by endometrial cup cells activates TGFB
}

\author{
Robert Kammerer ${ }^{1}$, Angela Ballesteros², Daniel Bonsor ${ }^{3}$, James Warren ${ }^{4}$, John M Williams ${ }^{5}$, \\ Tom Moore $^{5}$ and Gabriela Dveksler ${ }^{4}$ \\ ${ }^{1}$ Institute of Immunology, Friedrich-Loeffler-Institut, Greifswald-Insel Riems, Germany, ${ }^{2}$ Molecular Physiology and \\ Biophysics Section, National Institute of Neurological Disorders and Stroke, National Institutes of Health, Bethesda, \\ Maryland, USA, ${ }^{3}$ Institute of Human Virology, University of Maryland School of Medicine, University of Maryland, \\ Baltimore, Maryland, USA, ${ }^{4}$ Department of Pathology, Uniformed Services University, Bethesda, Maryland, USA and \\ ${ }^{5}$ School of Biochemistry and Cell Biology, University College Cork, Cork, Ireland
}

Correspondence should be addressed to R Kammerer or G Dveksler; Email: robert.kammerer@fli.de or gabriela.dveksler@usuhs.edu

\begin{abstract}
In early equine pregnancy, a highly invasive trophoblast cell subpopulation, the chorionic girdle cells, invade the endometrium and form endometrial cups (EC). These cells express classical MHC molecules, thereby stimulating a humoral and cellular immune response, resulting in a massive accumulation of maternal CD4+ and CD8+ T cells around the EC. Nevertheless, no immediate destruction of endometrial cups by maternal lymphoid cells occurs, presumably due to immune tolerance. Although the environment of EC is rich in TGFB and in FOXP3+, CD4+ T cells, the mechanisms leading to tolerance have not been elucidated. Recently, we discovered that equine trophoblast cells secrete pregnancy-specific glycoproteins (PSGs). Since human and murine PSGs activate latent TGFB, we hypothesized that equine PSGs may have a similar activity. We performed plasmon surface resonance experiments to show that equine PSG CEACAM49 can directly bind to the latency-associated peptide (LAP) of both TGFB1 and TGFB2. We then found that the binding of CEACAM49 leads to the activation of TGFB1 as determined by both ELISA and cell-based assays. Furthermore, the activation of TGFB is a unique function of PSGs within the human CEA family, because CEACAM1, 3, 5, 6, 8 do not activate this cytokine. This finding further strengthens the classification of CEACAM49 as an equine PSG. Based on our results, we hypothesize that activation of latent TGFB in the EC environment by equine PSGs secreted by invasive trophoblast cells, could contribute to the generation of regulatory T cells (Tregs) to maintain immune tolerance.

Reproduction (2020) 160 685-694
\end{abstract}

\section{Introduction}

Pregnancy-specific glycoproteins (PSGs) are secreted members of the carcinoembryonic antigen (CEA) family, which are expressed by trophoblast cells (Kammerer \& Zimmermann 2010, Rattila et al. 2019). In contrast, the second group of the human CEA family, the carcinoembryonic antigen-related cell adhesion molecules (CEACAMs) are primarily membraneanchored glycoproteins, which are widely expressed in different human tissues (Beauchemin \& Arabzadeh 2013). However, due to alternative splicing and proteolytic cleavage CEACAMs may be released from cells and reach the circulation (Naghibalhossaini \& Ebadi 2006, Beauchemin \& Arabzadeh 2013). While CEACAMs exist in all analyzed mammalian species, PSGs are found in mammals which have a hemochorial placenta, including primates (Streydio et al. 1988, Watanabe \& Chou 1988, Zhou \& Hammarstrom 2001), rodents (Chan et al. 1988, Rudert et al. 1992, Kromer et al. 1996) and microbats
(Kammerer et al. 2017). This suggests that the intimate contact of fetal trophoblast cells with maternal blood supports PSG evolution. However, more recently, we identified PSG-like CEACAMs in the horse (Aleksic et al. 2016, Missbach et al. 2018). From the 16 CEACAM1related genes in the horse, 8 are $P S G$-like genes, of which 4 were found to be expressed in equine trophoblast cells (Aleksic et al. 2016). mRNA encoding CEACAM49 was detected in conceptus tissues and in cultured chorionic girdle cells but was neither detected in cells from the uterus nor in maternal blood cells, thus showing a fetalcell specific expression pattern (Aleksic et al. 2016).

The horse has an epitheliochorial placenta, although a subset of equine trophoblast cells invade the endometrium, and form a unique structure called the endometrial cups (Allen et al. 1973, Antczak et al. 2013). T cells of the mare recognize but do not kill endometrial cup cells for about 100 days of pregnancy (de Mestre et al. 2010). This suggests that endometrial cup cells are protected from $\mathrm{T}$ cell attack by a yet 
unknown tolerance mechanism (de Mestre et al. 2010). Interestingly, endometrial cups are surrounded by a large number of lymphoid cells. These cells were found to be enriched in FoxP3-expressing regulatory $\mathrm{T}$ cells (Tregs) (de Mestre et al. 2010). Transforming growth factor beta (TGFB) plays a critical role in the generation and immunosuppressive function of Tregs (Hadaschik \& Enk 2015). Therefore, it is not surprising that a high content of TGFB was previously found in the placenta of different species, and specifically in the area surrounding the endometrial cups of the equine placenta (Lea et al. 1995, Lennard et al. 1995).

TGFB is a member of a gene family composed of 33 genes in mammals (Morikawa et al. 2016). Three of them belong to the TGFB subgroup (TGFB1, TGFB2, $T G F B 3$ ) and all are synthesized as a precursor protein, consisting of a signal peptide, a prodomain and the mature polypeptide (Derynck \& Budi 2019). After cleavage of the signal peptide, the polypeptide is further processed by proteases to separate the prodomain (termed latency-associated polypeptide (LAP)) from the mature polypeptide, which remains non-covalently associated (Derynck \& Budi 2019). While TGFB homodimers are associated with LAP they cannot bind to their receptors and exist in a latent/inactive state termed the small latent complex (SLC) that is secreted from cells or binds to the plasma membraneassociated glycoprotein-A repetitions predominant (GARP) (Stockis et al. 2009). The SLC can also bind to the latent TGFB binding proteins (LTBPs) forming the large latent complex (LLC), which then binds to extracellular matrix (Robertson et al. 2015). In either case, TGFB has to be activated to be able to bind to its receptor (Shi et al. 2011, Lienart et al. 2018). The TGFB receptor complex is formed by oligomerization of two type 2 serine/threonine kinase receptors (TBR2) and two type I receptors (TBR1) (Derynck \& Budi 2019). Activation of the TGFB receptor leads to SMAD protein translocation to the nucleus and induction of their target genes transcription (Derynck \& Budi 2019). Therefore, activation of latent TGFB has been implicated as a major regulatory mechanism of TGFB function (Annes et al. 2003). Different mechanisms for latent TGFB activation have been described, including extremes of $\mathrm{pH}$, proteases, and surface integrins (Robertson \& Rifkin 2016). Which mechanisms are important for TGFB activation in the placenta or around EC remain unknown.

We found that members of the human and murine PSG family, which are expressed in increasing concentrations as pregnancy progresses, can activate latent TGFB1 (Moore \& Dveksler 2014, Ballesteros et al. 2015, Warren et al. 2018). This is of interest, since PSGs are highly expressed by syncytiotrophoblast cells which represent the fetal frontline at the feto-maternal interface. Thus, the fetus by itself may regulate the availability of active TGFB in its immediate vicinity.
This may be of particular relevance with respect to the immune suppressive function of TGFB, which may lead to the generation of Tregs at the feto-maternal interface. Even more intriguing is the finding that human and murine PSGs share the ability to activate TGFB, despite their different structures and their independent evolution (Warren et al. 2018). This indicates that TGFB activation is a pivotal function of PSGs.

Therefore, we hypothesized that activation of TGFB in the human is specific to PSGs within the CEA family and that, PSGs from other species, such as the horse, may also be able to activate latent TGFB. Indeed, we show in this report that human PSG1 binds to LAP of TGFB1 and TGFB2 and that this interaction is PSG-specific, since none of the human CEACAMs (CEACAM1, 3, 5, 6, 8) can bind to LAP of TGFB1 or TGFB2. We further demonstrate that the equine PSG, CEACAM49, can activate latent TGFB, like human PSGs. Given the fact that TGFB is abundant at EC and the leucocyte infiltrate demarking the EC contains a large amount of FoxP3 expressing T cells, our findings support the hypothesis that equine EC cells control the activity of maternal immune cells by regulating the availability of active TGFB through a PSGdependent mechanism.

\section{Materials and methods}

\section{Generation and purification of recombinant proteins}

CEACAM49 and PSG1-V5His production and purification were performed as previously described (Shanley et al. 2013), using transient transfection of HEK-293 cells (Freestyle ${ }^{\text {TM }}$ 293 cells) with CEACAM49 and PSG1 coding sequences (CDS) cloned into the pTT3 expression vector. The fulllength cDNA of CEACAM49 was cloned from endometrial cup tissues, as previously described (Aleksic et al. 2016). The pTT3-CC49 construct was generated by PCR amplifying the CEACAM49 CDS from pRc/CMV-ecaCEACAM49 incorporating 5' ECORI and 3' HindIII restriction sites using the primers, ecaCEACAM49For: 5'-gGAATTCaccatgcaatcaccctca and ecaCEACAM49Rev: 5'-cAAGCTtggctctgtaactgggg. The PCR amplified band was blunt cloned into PSTBlue1 and sub-cloned into pTT3 using EcoRI and HindIII restriction enzymes, in frame with a V5-His tag as previously described (Shanley et al. 2013). Recombinant proteins were purified from cell culture medium $72 \mathrm{~h}$ post-transfection using a Qiagen Ni-NTA resin. Imidazole (Sigma,) was added to the culture medium to a final concentration of $10 \mathrm{mM}$ to reduce nonspecific binding. Ni-NTA resin was added to the medium at a ratio of $1 \mathrm{~mL}$ resin suspension (corresponding to $0.5 \mathrm{~mL}$ resin bed volume) to $100 \mathrm{~mL}$ medium. The medium and resin were then batch bound overnight on a rotating wheel at $4^{\circ} \mathrm{C}$. The medium and resin mix was then passed through a disposable polypropylene column (Thermo Fisher Scientific) and the resin was washed with $500 \mathrm{mM} \mathrm{NaCl}, 20 \mathrm{mM} \mathrm{NaH}{ }_{2} \mathrm{PO}_{4}$, pH6 (wash buffer). Protein was then eluted from the column with increasing concentrations of imidazole in wash buffer. Fractions containing recombinant PSG1 or CEACAM49 were then pooled and passed through a new column containing 
$0.5 \mathrm{~mL}$ Ni-NTA resin, the flow-through collected, and bound protein eluted with $1 \mathrm{~mL} 50 \mathrm{mM}$ imidazole followed by three $1 \mathrm{~mL} 200 \mathrm{mM}$ imidazole fractions. The flow-through and three $200 \mathrm{mM}$ fractions were pooled and concentrated to a volume of $1 \mathrm{~mL}$ using a Millipore Amicon Ultra Ultracel 10K centrifugal filter (Millipore). For size exclusion chromatography (SEC) 1 $4 \mathrm{mg}$ of purified recombinant protein was applied to a HiLoad 16/60 Superdex S200 prep grade column in a $1 \mathrm{~mL}$ volume with a flow rate of $1 \mathrm{~mL} / \mathrm{min}$ using the AKTA explorer system in PBS. Determination of the $1 \mathrm{~mL}$ fractions to pool was based on chromatogram peaks and the resultant protein solution was further concentrated.

Human LAP of TGFB1 and LAP of TGFB2 were synthesized as a gene string (Thermo Fisher Scientific) and cloned directly into HindIII/Notl cut pcDNA4.0 (Thermo Fisher Scientific). The extracellular domains of CEACAM5 was PCR amplified from HG11077 CH (Sino Biological) and cloned into Xmal/Notl cut pSG160 (Bonsor et al. 2015). The three proteins had a $6 x \mathrm{His}$ tag at the C-terminus and were expressed in HEK cells grown in FreeStyle 293 supplemented with GlutaMAX and gentamicin (Thermo Fisher Scientific) by transfections of the plasmids with polyethyleneimine. Cells were grown for 5 days at $37^{\circ} \mathrm{C}$ with $8 \% \mathrm{CO}_{2}$. The proteins were directly purified from the clarified supernatant using a 5-mL HisTrap Excel column and further purified on a Superdex 200 column (GE Healthcare) equilibrated with $20 \mathrm{mM}$ Tris, $150 \mathrm{mM} \mathrm{NaCl}$, pH 7.5. Recombinant CEACAMs were generated as previously described with minor modifications (Singer et al. 2014). Plasmids encoding CEACAM1, CEACAM3, CEACAM6 and CEACAM8 containing an Fc-tag at their C-terminus were generously provided by Esther Klaile (University Hospital Jena, Germany) and Bernhard Singer (University of Essen, Germany). The plasmids were transfected into Expi293 cells following the manufacturer's recommendations and the supernatants were harvested 6 days post-transfection (Thermo Fisher Scientific). PSG1-Fc was generated from the supernatant of a stably transfected $\mathrm{CHO}$ $\mathrm{K} 1$ single-cell clone established in our laboratory that was grown in a hollow fiber cartridge bioreactor (FiberCell Systems, Frederick, MD, USA) as previously described (Ballesteros et al. 2015). The Fc control protein which comprises the $\mathrm{CH} 2$ and $\mathrm{CH} 3$ domains of the human IgG1 heavy chain and the hinge region was generated by transfecting the pFuse-hlgG1e3-Fc2 plasmid (InvivoGen, CA, USA) into ExpiCHO cells following the manufacturer's recommendations for the high titer protocol and the supernatants were harvested 10 days post-transfection (Thermo Fisher Scientific). Clarified supernatants were loaded onto a MabSelect protein A column (GE Healthcare) equilibrated with PBS and the proteins were eluted with $0.1 \mathrm{M}$ glycine $\mathrm{pH}$ 2.7 and neutralized immediately with $1 \mathrm{M}$ Tris buffer $\mathrm{pH}$ 8.0. All proteins were buffer exchanged into PBS and concentrated using Amicon Ultra-15 10K MW cut off centrifugal filter units (Millipore).

All expressed CEACAMs are composed only of the extracellular domains. The transmembrane and the cytoplasmic tails or the GPI linkage sequences are not included in the cDNAs utilized to express these proteins.

Protein concentrations were determined by absorbance at 280 $\mathrm{nm}$ with adjustment for their respective extinction coefficients, following their separation on NuPAGE 4-12\% Bis Tris gels with
MOPS running buffer and visualization with GelCode Blue. The proteins were prepared by adding NuPAGE LDS sample buffer and sample reducing agent, which contains DTT. All of these reagents were purchased from Thermo Fisher Scientific.

\section{Activation of the small latent complex (SLC) of TGFB1 by PSGs}

To detect active TGFB1, we performed two assays as previously described using triplicate samples for each condition tested (Ballesteros et al. 2015). In the cell-free system assay, proteins were incubated with $50 \mathrm{ng} / \mathrm{mL}$ of SLC (R\&D Systems) at $37^{\circ} \mathrm{C}$ for $1 \mathrm{~h}$ in a final volume of $0.1 \mathrm{~mL}$ of PBS in siliconized tubes. The samples were then transferred to wells of a 96-well Nunc Maxisorb plate that had been coated overnight with recombinant human TGFB receptor 2-FC (R\&D systems) and blocked with PBS-1\% BSA. After 2 h, the plates were washed, and active TGFB1 was detected with a biotinylated antibody specific for mature TGFB1 (BAF240, R\&D systems). In the second assay, bioactive TGFB was determined using the TGFB responsive plasminogen activator inhibitor-1 reporter mink lung epithelial cells line (TMLEC) provided by Dr Rifkin (New York University, NY, USA) following incubation of the proteins with $50 \mathrm{ng} / \mathrm{mL}$ of $\mathrm{SLC}\left(\mathrm{R} \& \mathrm{D}\right.$ Systems) at $37^{\circ} \mathrm{C}$ for $1 \mathrm{~h}$ in a final volume of $0.1 \mathrm{ml}$ of serum-free DMEM supplemented with $0.1 \%$ insulin-transferrin-sodium selenite (ITS) (Sigma-Aldrich) in siliconized tubes, which was then added to the cells for 16-18 h (Abe et al. 1994). After this time, the cells were lyzed and luciferase was measured in a GloMax Discover system (Promega). To verify that the observed increase in luciferase was the result of TGFB binding to its receptor in the TGFB reporter cells, the experiments were carried out in the presence of 5 $\mu \mathrm{M}$ of the TGFB receptor 1 kinase inhibitor SB431542 (R\&D Systems) as previously described (Blois et al. 2014). Two-fold dilutions of active TGFB1 (R\&D Systems) from 2000 to 62.5 $\mathrm{pg} / \mathrm{mL}$ were used as standards to determine the linearity of the signal in these assays. Controls included SLC incubated with the same volume of PBS (vehicle) or a protein consisting of the Fc portion of human IgG1, which undergoes glycosylation due to the presence of two $\mathrm{N}$-glycosylation sites. We observed no differences between these two controls in any of our assays.

\section{Binding of CEACAMs and PSG1 to LAP-TGFB1 and LAP-TGFB2}

For LAP binding assays, wells (Nunc Maxisorb) were coated overnight with either recombinant human LAP-TGFB1 or LAPTGFB2 and blocked with PBS-2\% BSA. The wells were washed three times (PBS/0.05\% Tween-20) and the samples to be tested were added and incubated for $4 \mathrm{~h}$ at room temperature. The wells were washed again and incubated with HRP-labeled anti-human Fc antibody (Thermo Fisher Scientific) for $1 \mathrm{~h}$. After three final washes, binding of the antibody was visualized with TMB substrate.

Surface plasmon resonance (SPR) experiments were performed in a BIAcore 3000 instrument (GE Healthcare) at a flow rate of $10 \mu \mathrm{L} / \mathrm{min}$ and $25^{\circ} \mathrm{C}$ using HBS-EP $(0.01 \mathrm{M}$ HEPES pH 7.4, $0.15 \mathrm{M} \mathrm{NaCl}, 3 \mathrm{mM}$ EDTA, and $0.005 \%$, v/v 
Surfactant P20) as running buffer. Purified recombinant LAP of TGFB2 or LAP of TGFB1 diluted in $10 \mathrm{mM}$ sodium acetate $\mathrm{pH} 4.0$ buffer were immobilized onto a CM5 sensor chip (GE Healthcare) using standard amine coupling chemistry. A control surface where no protein was immobilized was used as control to correct for background and potential non-specific binding. PSG1 or CEACAM49 were injected over a range of concentrations $(20 \mu \mathrm{M}$ to $30 \mathrm{nM})$ for $180 \mathrm{~s}$ followed by a 240 $\mathrm{s}$ dissociation period. To regenerate the surface after each protein injection, a solution containing $10 \mathrm{mM}$ Glycine- $\mathrm{HCl}$ $\mathrm{pH} 2.0$ was injected for $30 \mathrm{~s}$. Real-time data were analyzed using the BIAevaluation 4.1 software (GE Healthcare), and kinetic profiles were fitted using a global 1:1 binding algorithm to estimate the association (ka) and dissociation (kd) constant, and the affinity (KD) of the interaction. Drift correction was added for the interactions of CEACAM49 with LAP of TGFB1 (maximum drift of 0.052 RU) and LAP of TGFB2 (maximum drift of $0.103 \mathrm{RU}$ ) to correct for baseline drift. Realtime sensorgrams were aligned using the BIAevaluation 4.1 software (GE Healthcare) and final figures were generated in Prism8 (GraphPad).

\section{Phylogenetic analyses and datasets}

Phylogenetic analyses based on nucleotide and amino acid sequences were conducted using MEGA X (Kumar et al. 2018). Sequence alignments were performed using Muscle implemented in MEGA X. Phylogenetic trees were constructed using the maximum likelihood $(\mathrm{ML})$ method with bootstrap testing (500 replicates) and the Tamura-Nei substitution model. The following Ensembl releases were used to analyze CEACAM loci: human, GRCh38.p13; mouse, GRCm.p6; horse, EquCab3.

\section{Statistical analysis}

Activation of latent TGFB1 is reported as the mean fold increase over control \pm S.E. One-way ANOVA and the post hoc Tukey tests for pairwise comparisons were used for statistical analysis. Data groups passed the Shapiro-Wilk test for normality and the Brown-Forsythe test for equal variances. Significant differences between binding of PSG1-Fc and control to LAP of TGFB1 or LAP of TGFB2 were calculated using the Student's t-test. Data were analyzed using version 8 of GraphPad Prism (GraphPad Software) and a $P$ value of $<0.05$ was considered statistically significant.

\section{Ethics approval and consent to participate}

In the present study, we did not use human or animal tissues. In addition, we did not perform animal experiments.

\section{Results}

\section{Activation of TGFB1 is specific for PSGs within the human CEA family}

We have previously shown that all human PSGs activate latent TGFB1 and that this activity is preserved whether the protein has an Fc or a V5His tag as observed with

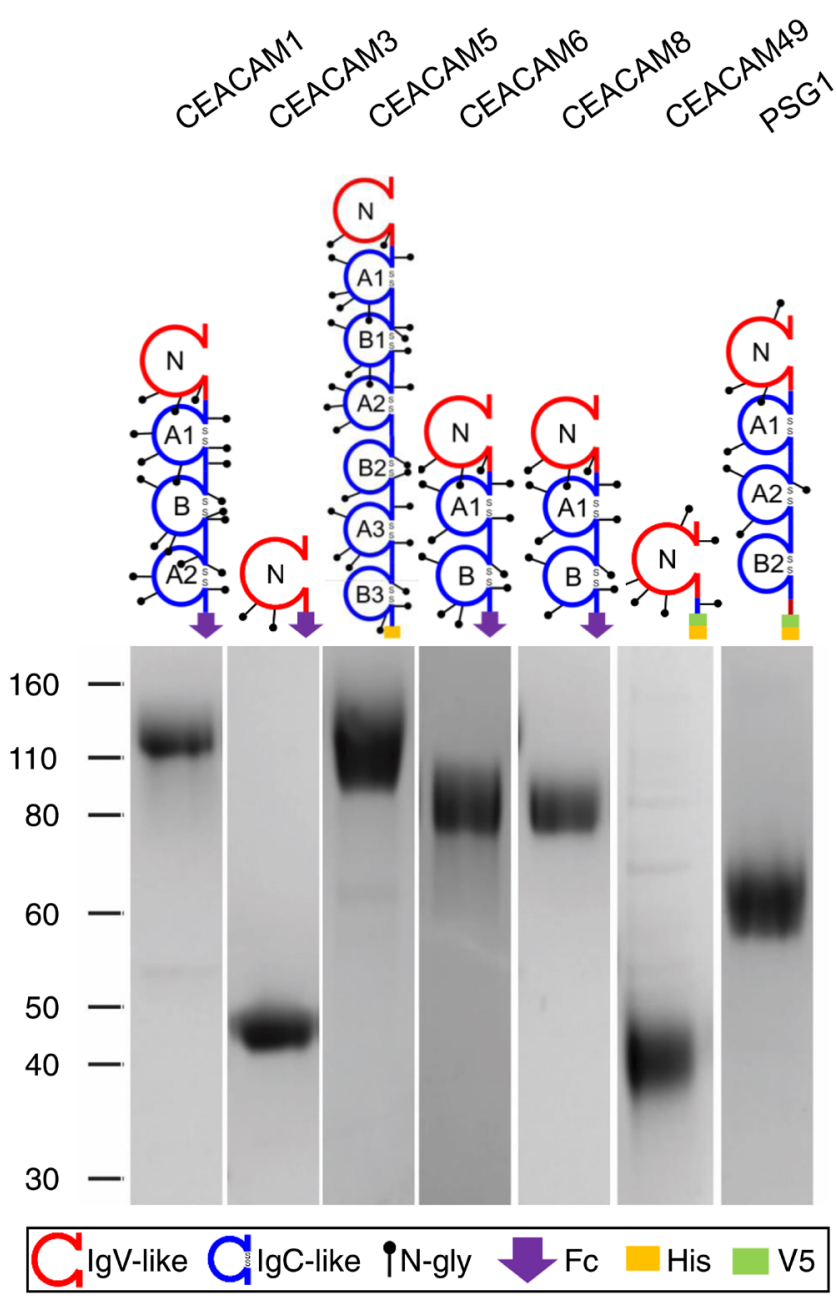

Figure 1 Generated recombinant CEACAMs and PSG1. The proteins with their different tags are schematically shown above the gel. Recombinant CEACAM1-Fc, CEACAM3-Fc, CEACAM5-His, CEACAM6-Fc, CEACAM8-Fc, CEACAM49-V5His, and PSG1-V5His were separated on $4-12 \%$ SDS-PAGE gels under denatured and reducing conditions and stained with GelCode blue.

PSG1 (Blois et al. 2014, Warren et al. 2018). In addition, we observed that while more than one PSG domain can activate latent TGFB1, the B2 domain is the most efficient (Ballesteros et al. 2015). Since B domains are also found in human CEACAMs we wondered whether human CEACAMs can also activate latent TGFB1. Therefore, we expressed recombinant human CEACAMs with 6xHis (CEACAM5) or an FC-tag (CEACAM1, 3, 6 and 8 ) and compared their activity to PSG1 with a $6 x \mathrm{His}$ and a V5 tag at the C-terminus. The purity of all proteins was confirmed by staining with GelCode Blue following separation on $4-12 \%$ SDS-PAGE gels (Fig. 1).

We then used these proteins to test their ability to activate latent TGFB1 using two different assays: an ELISA (Fig. 2A) and a cell-based assay (data not shown). Both assays gave qualitatively similar results. As shown in Fig. 2A, only PSG1 significantly activated TGFB1, 

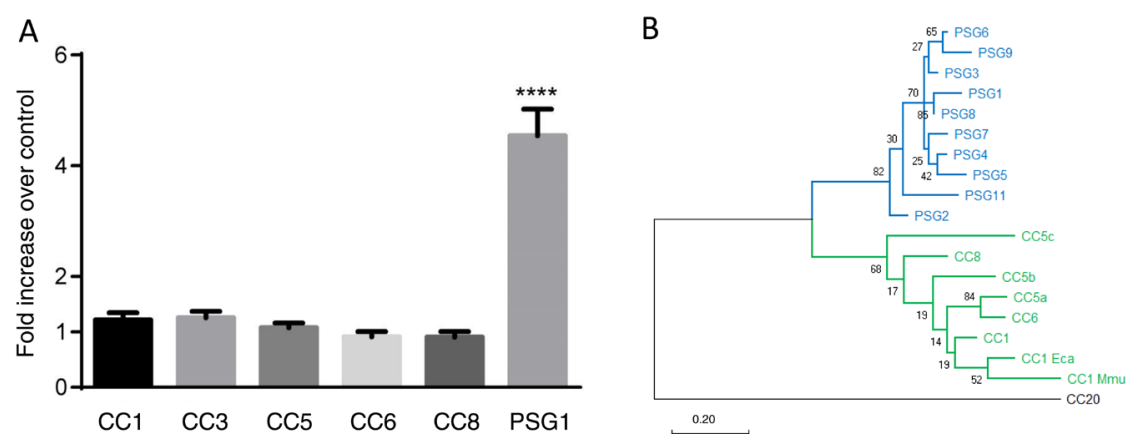

Figure 2 Activation of latent TGFB1 by members of the human CEACAM family and by human PSG1. (A) $280 \mathrm{nM}$ of CEACAM (CC) 1, $3,5,6$ and 8 or PSG1 were incubated with 50 $\mathrm{ng} / \mathrm{mL}$ of SLC for $1 \mathrm{~h}$ at $37^{\circ} \mathrm{C}$ in PBS. The concentration of active TGFB1 was determined as indicated in the materials and methods section and expressed as fold activation over Fc protein control designated as 1 . Statistical analysis was performed with one-way ANOVA followed by Dunnet's pairwise test. ${ }^{* * * *} P<$ $0.0001(n=3)$. (B) Phylogenetic analysis of human PSG B domains and selected human, murine and equine CEACAM $B$ domains were performed using amino acid sequences, the $\mathrm{ML}$ method and the MEGA X software. CC1 Eca, CEACAM1 from horse (Equus caballus); CC1 $\mathrm{Mmu}, \mathrm{CEACAM} 1^{\mathrm{a}}$ from mouse (Mus musculus). but although some CEACAMs have B domains (Fig. 1), none of the CEACAMs was able to activate latent TGFB1 at the concentrations tested (Fig. 2A). Most strikingly, even CEACAM5 which has three $B$ domains did not reproducibly activate latent TGFB1. This suggests that fundamental differences exist between B domains of human PSGs and CEACAMs. Indeed, phylogenetic analysis showed that the amino acid sequences of PSG $B$ domains and the $B$ domains of human, murine and equine CEACAMs were clearly separated with each forming an individual cluster. The cluster of human PSG sequences has relatively short branches compared to the branches in the CEACAM cluster, indicating either concerted evolution of PSG $\mathrm{B}$ domains or a more recent PSG duplications compared to CEACAMs (Fig.2B).

\section{Equine PSG CEACAM49 binds to LAP of TGFB1 and TGFB2}

We reported that human and murine PSGs interact with LAP of TGFB1 and that human PSGs use the IgC-like $\mathrm{B}$ domain to bind to LAP while murine PSGs used the $\mathrm{N}$ domain (Ballesteros et al. 2015, Warren et al. 2018). Equine PSGs are extremely small consisting of only a single IgV-like domain (Aleksic et al. 2016), thus the interaction could only take place via the $\mathrm{N}$ domain similar to the interaction of latent TGFB1 with murine PSGs. Previously, we showed that PSG1 binds to the commercially available LAP-TGFB1 (Ballesteros et al. 2015). Now, we asked if human PSG1 can also bind to LAP-TGFB2. Because LAP-TGFB2 is not commercially available, we generated this protein for binding studies. Plates were coated with LAP-TGFB1 or LAP-TGFB2 and incubated with PSG1-Fc or the control Fc-fusion protein (Ballesteros et al. 2015). PSG1-Fc binds to both LAPTGFB1 and LAP-TGFB2 while the control Fc protein did not, demonstrating that human PSG1 can bind not only to LAP-TGFB1 as previously observed but also to LAPTGFB2 (Fig. 3A). We performed similar experiments with the other CEACAMs with an Fc-tag (CC1, CC3, CC6 and
CC8). Consistent with their inability to activate TGFB described previously, none bound to LAP-TGFB1 and LAP-TGFB2 over the FC control protein (data not shown). Because some of the proteins we generated have a His tag rather than the Fc-tag, we could not utilize ELISA to study their interaction as the His tag is also present in LAP-TGFB2. Therefore, we studied the potential binding with the LAP peptides using surface plasmon resonance, which also allowed us to determine the kinetics and the affinity of the interactions.

We generated a biosurface with immobilized LAPTGFB1 or LAP-TGFB2 and injected CC49 or PSG1 for $3 \mathrm{~min}$. After a dissociation phase of $3 \mathrm{~min}$, the LAP surfaces were regenerated with glycine buffer to reach the baseline level and start a subsequent experimental cycle as indicated in Material and Methods and depicted in Fig. 3B. We first analyzed the binding affinity of human PSG1V5His to LAP-TGFB2 using a Biacore 3000 instrument (GE Healthcare). As shown in Fig. 3E and Table 1, PSG1$\mathrm{V} 5 \mathrm{His}$ binds to LAP-TGFB2 with an affinity of $100 \mathrm{nM}$, which is about 40 times higher than the affinity of this protein for the LAP-TGFB1 (Warren et al. 2018). Next, we tested if CEACAM49 interacts with LAP of either TGFB1 or TGFB2. CEACAM49 binds to both LAP peptides in a micromolecular range with a KD of $10 \mu \mathrm{M}$ to LAP-TGFB1 and a KD of $26 \mu \mathrm{M}$ to LAP-TGFB2 (Fig. 3B, C, D and Table 1). Thus, CEACAM49 binds with a slightly lower affinity to the LAP peptides than previously determined for the interaction of human PSG1 and TGFB1 (Table 1). The half-lives of CEACAM49-LAP $\beta 1$ and CEACAM49-LAP $\beta 2$ complexes was $67.6 \mathrm{~min}$ and $71.8 \mathrm{~min}$, respectively while human PSG1 forms complexes with TGFB2 with a half-life of 14.4 min (Table 1).

\section{Equine CEACAM49 activates TGFB1}

After demonstrating that CEACAM49 binds to LAP-TGFB1 with similar binding characteristics as human PSG1, we investigated if CEACAM49 is also able to activate the latent TGFB1. To evaluate this, we incubated SLC (LAP- 


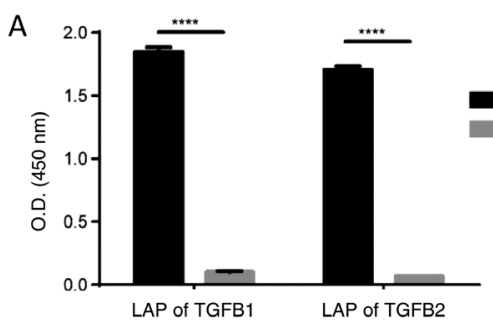

B

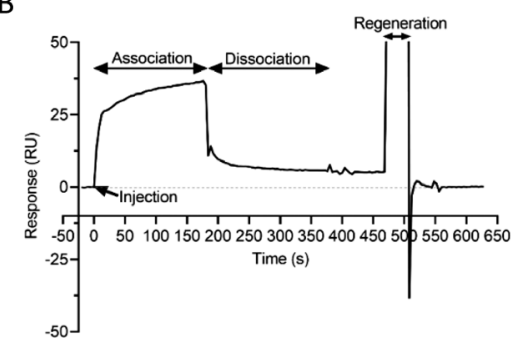

D Equine CEACAM49 - LAP of TGFB2

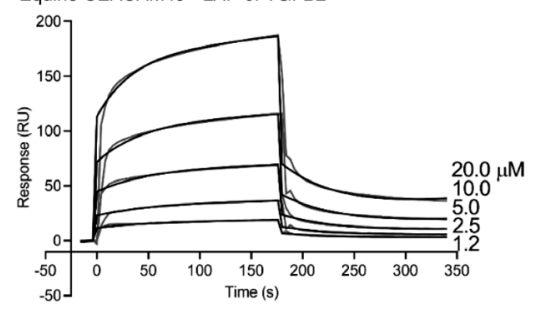

Fc
Human PSG1-FC

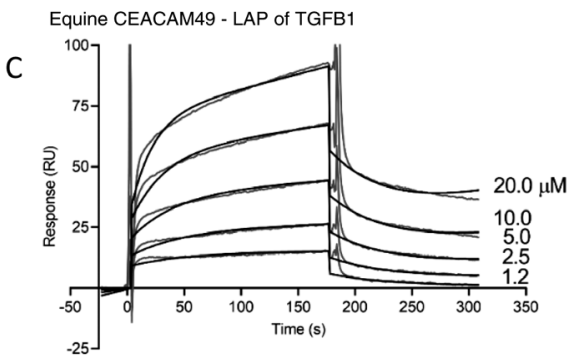

E

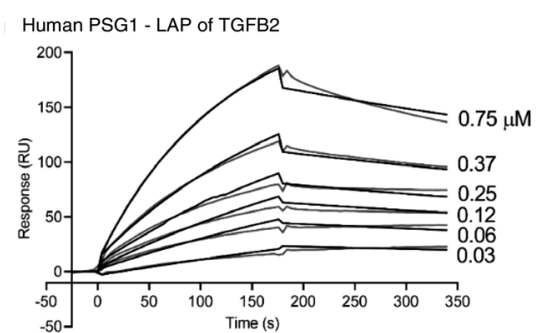

Figure 3 Binding of CEACAM49 and PSG1 to the LAP of TGFB1 and LAP of TGFB2. (A) PSG1 or FC control protein were added to wells previously coated with LAP of TGFB1 or LAP of TGFB2. (B) Representative SPR sensorgram of the interaction of CC49 at 0.2 $\mathrm{mM}$ with immobilized LAP of TGFB2. The beginning of the CC49 injection, association, dissociation, and regeneration phases are shown. A surface without immobilized protein was used as a control for potential nonspecific binding to the biosensor surface and was subtracted from the binding of CC49 to the LAP of TGFB2 coated surface. (C) Sensorgrams of the interaction of CC49 with the LAP of TGFB1. Serial dilutions of CC49 ranging from 20.0 to $1.2 \mathrm{mM}$ were injected during 3 min over a CM5 biosensor chip with immobilized LAP of the TGFB1. SPR sensorgrams for each protein concentration are shown as grey lines while the fitted data are shown as black lines. (D) Sensorgrams of the interaction of CC 49 with the LAP of the TGFB2 represented as in B. (E) Sensorgram of the interaction of PSG1 with the LAP of the TGFB2 represented as in $B$. The specific concentration utilized for each sensorgram is indicated. ${ }^{* * * *} P<0.0001$
TGFB1) with CEACAM49-V5His for $1 \mathrm{~h}$ at $37^{\circ} \mathrm{C}$ and then determined the amount of activated TGFB1 using an ELISA-based protocol with the TGFB receptor II as capture as previously described (Blois et al. 2014, Ballesteros et al. 2015, Warren et al. 2018). We found that CEACAM49 activated the SLC of TGFB1 in a concentration-dependent manner (Fig. 4A). In previous experiments, PSG1 at $140 \mathrm{nM}$ induces approximately a 1.5 increase over the control in the luciferase signal in the luciferase reporter assay (Warren et al. 2018). To confirm these results, we also performed a cell-based functional assay. Again, we observed a concentration-dependent activation of the SLC of TGFB1 as indicated by the increase in luciferase expression in the reporter cells (Fig. 4B). To demonstrate the specificity of the reaction, we incubated the cells with the TGFB1 receptor I inhibitor SB431542 or the vehicle control (DMSO) together with CEACAM49. The increase in luciferase activity, which is readout of active TGFB signaling in these reporter cells, was blocked in the presence of the TGFB receptor I inhibitor, while the vehicle control had no effect (Fig. 4C). These experiments strongly suggest that CEACAM49 not only binds to the SLC but also activates TGFB. Thus human, murine and equine PSGs function as TGFB activators. Unfortunately, because the SLC of TGFB2 is not commercially available and we were unable to generate it, we could not perform these experiments with latent TGFB2.

\section{Synthenic relationship and exon composition suggest convergent evolution of equine, murine and human PSGs}

Given the similar functionalities of equine PSGs with murine and human PSGs (this study (Aleksic et al.

Table 1 Kinetics constants and affinity of the interaction of CEACAM49 (CC49) with the LAP of TGFB1 and of TGFB2 and of PSG1 with the LAP of TGFB2.

\begin{tabular}{|c|c|c|c|c|c|c|}
\hline Interaction & $\mathbf{k a} / \mathbf{M} / \mathbf{s}($ mean \pm S.D. $)$ & kd/s (mean \pm S.D.) & $\mathbf{K D}(\mu \mathrm{M})$ & $\boldsymbol{R} \boldsymbol{m a x}($ mean \pm S.D. $)$ & chi $^{2}$ & $\mathbf{t} 1 / 2(\min )$ \\
\hline CC49 - LAP-TGFB1 & $(1.7 \pm 0.06) \times 10^{3}$ & $(1.7 \pm 0.7) \times 10^{-4}$ & 10 & $55.2 \pm 1.3$ & 3.3 & 67.6 \\
\hline CC49 - LAP-TGFB2 & $(0.6 \pm 0.03) \times 10^{3}$ & $(1.6 \pm 0.5) \times 10^{-4}$ & 26 & $129.0 \pm 4.8$ & 1.2 & 71.8 \\
\hline PSG1 - LAP-TGFB2 & $(8.8 \pm 0.10) \times 10^{3}$ & $(0.8 \pm 0.1) \times 10^{-3}$ & 0.1 & $268.0 \pm 3.6$ & 3.8 & 14.4 \\
\hline *PSG1 - LAP-TGFB1 & $(1.4 \pm 0.30) \times 10^{3}$ & $(6.1 \pm 0.4) \times 10^{-3}$ & 4.4 & $44.2 \pm 0.5$ & 3.0 & 1.9 \\
\hline
\end{tabular}

The SPR sensorgrams shown in Fig. 3C, D and E for the interaction of CEACAM49 with the LAP of TGFB1 and of TGFB2 and of PSG1 with the LAP of TGFB2 were fitted using a 1:1 Langmuir binding model for the estimation of the association and dissociation rates (ka and kd, respectively), and affinity $(K D=k a / k d)$. The maximum response $(R m a x), \chi^{2}\left(c^{2} i^{2}\right)$ values for the fitted data and the complex half-time life $\left(t_{1 / 2}\right)$ are also indicated.

* Published in Warren et al. (2018). 

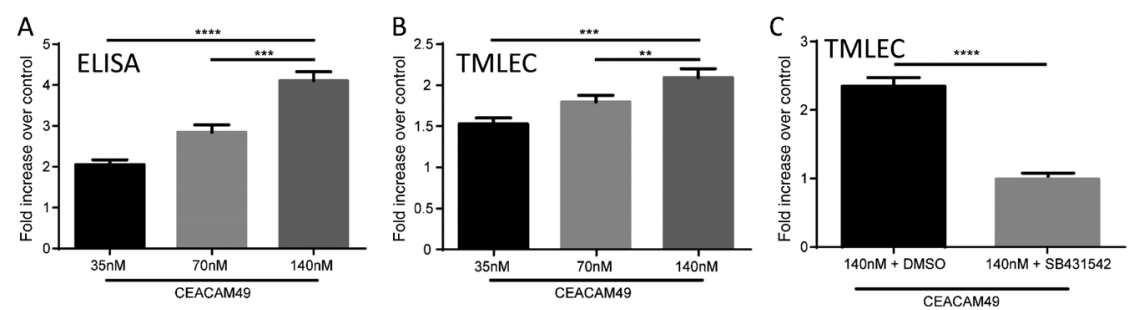

Figure 4 CEACAM49 activates the small latent
complex (SLC) of TGFB1. (A) CEACAM 49 at the
indicated concentrations or PBS were
incubated with SLC for $1 \mathrm{~h}$ at $37^{\circ} \mathrm{C}$ in PBS. The
TGFB1 concentration was determined by ELISA
and represented as fold increase over the PBS
control. All treatments were made in triplicate
wells. (B) CEACAM 49 at the indicated
concentrations or PBS (control) were incubated
with SLC for $1 \mathrm{~h}$ at $37^{\circ} \mathrm{C}$ in DMEM- $0.1 \%$ ITS
and then added to TMLEC reporter cells. The
treated TMLECs were incubated for $18 \mathrm{~h}$ and
then lysed. The luciferase activity in the lysate
was analyzed with a GloMax luminometer. (C)
The TMLEC assay described in A was performed
in the presence of the specific TGFB receptor I
inhibitor SB 431542 or DMSO (vehicle control).
$* * P \leq 0.01 ; * * * P \leq 0.001 ; * * * * P \leq 0.0001$.

2016)), the question arises as to whether a common ancestor of equine PSGs with human or rodent PSGs can be inferred. Comparison of the loci of the CEA gene families in equine and murine genomes suggests that PSGs evolved independently in horses and mice, since the location of equine PSGs is close to the CEACAM genes, similar to human PSG genes, and not in a separated locus as murine Psg genes, which are located between Pglyrp1 and Npas 1 (Fig. 5A). Next, we compared the exon arrangement of equine PSG genes with that of human PSG genes (Fig. 5B). Equine PSG genes are composed of a single IgV-like domain exon and a single IgC-like domain exon. The IgC-like domain exon is similar to the $\mathrm{A} 2$ exon of CEACAM1. In contrast, human PSG genes have three IgC-like domain encoding exons. Two of them have sequence similarity with the A1 exon of CEACAM1 and one has similarities to the B-domain encoding exon of CEACAM1. Thus, equine and human PSG genes contain distinct IgC-domain exons which suggest that no common ancestor of equine and human PSGs existed (Fig. 5B).

\section{Discussion}

In this report, we show that the recently identified equine PSG CEACAM49 can directly bind to LAP of TGFB1 and TGFB2 with similar affinity and binding parameters resulting in a half-life time of the CEACAM49-LAP complex of approximately $1 \mathrm{~h}$. The affinity of CEACAM 49 for LAP of TGFB1 is in the same range as previously found for human PSGs (Ballesteros et al. 2015, Warren et al. 2018). On the other hand, the affinity of the interaction of CEACAM49 with LAP of TGFB2 is 250 times weaker than that of PSG1 mainly due to the slower association observed with CEACAM49. We further demonstrate by ELISA and a cell-based functional assay that binding of CEACAM49 to the SLC leads to TGFB1 activation. In the experiments presented, we used human LAP of TGFB1 and TGFB2 and the small latent complex of human
TGFB1 as their equine counterparts are not available. It is important to note that equine TGFB1 and TGFB2 have a $92 \%$ and $99 \%$ identity to their human counterparts, respectively.

The ability to activate latent TGFB is a PSG-specific function within the human CEA family, since none of the tested human CEACAMs were able to activate TGFB1. This indicates that activation of latent TGFB is a newly acquired function for the CEA family following the evolution of PSGs.

Surprisingly, there is no evidence suggesting that a common ancestor of PSGs from different mammalian lineages exist. In contrast, comparison of the PSG gene locus and the exon composition suggest that PSGs evolved independently in various mammalian lineages, although further analysis is needed to understand the evolution of PSGs in different mammalian lineages. If PSGs evolved independently at least three times in mammals, common functions of PSGs in different species are the result of convergent evolution.

Convergent evolution requires a strong selective pressure (Laayouni et al. 2014); therefore, our finding indicates that the regulation of TGFB activity is a key function of PSGs. Remarkably, the TGFB superfamily is a master regulator in the placenta, playing pivotal roles in tissue remodeling, trophoblast invasion, neovascularization as well as in the regulation of immune responses (Jones et al. 2006). In addition to TGFB activation, we have previously identified the inhibition of platelet aggregation to be a common function of human, murine and equine PSGs (Shanley et al. 2013, Aleksic et al. 2016). Moreover, at least one human and murine PSG were found to play a role in angiogenesis (Lisboa et al. 2011, Blois et al. 2012) as well as in the induction of immunosuppressive cytokines including TGFB by immune cells such as monocytes and dendritic cells (Motran et al. 2003, Martinez et al. 2012, Falcon et al. 2014). All of these activities attributed to PSGs, together with the activation of TGFB, contribute to ensure a successful pregnancy that may provide the 
A

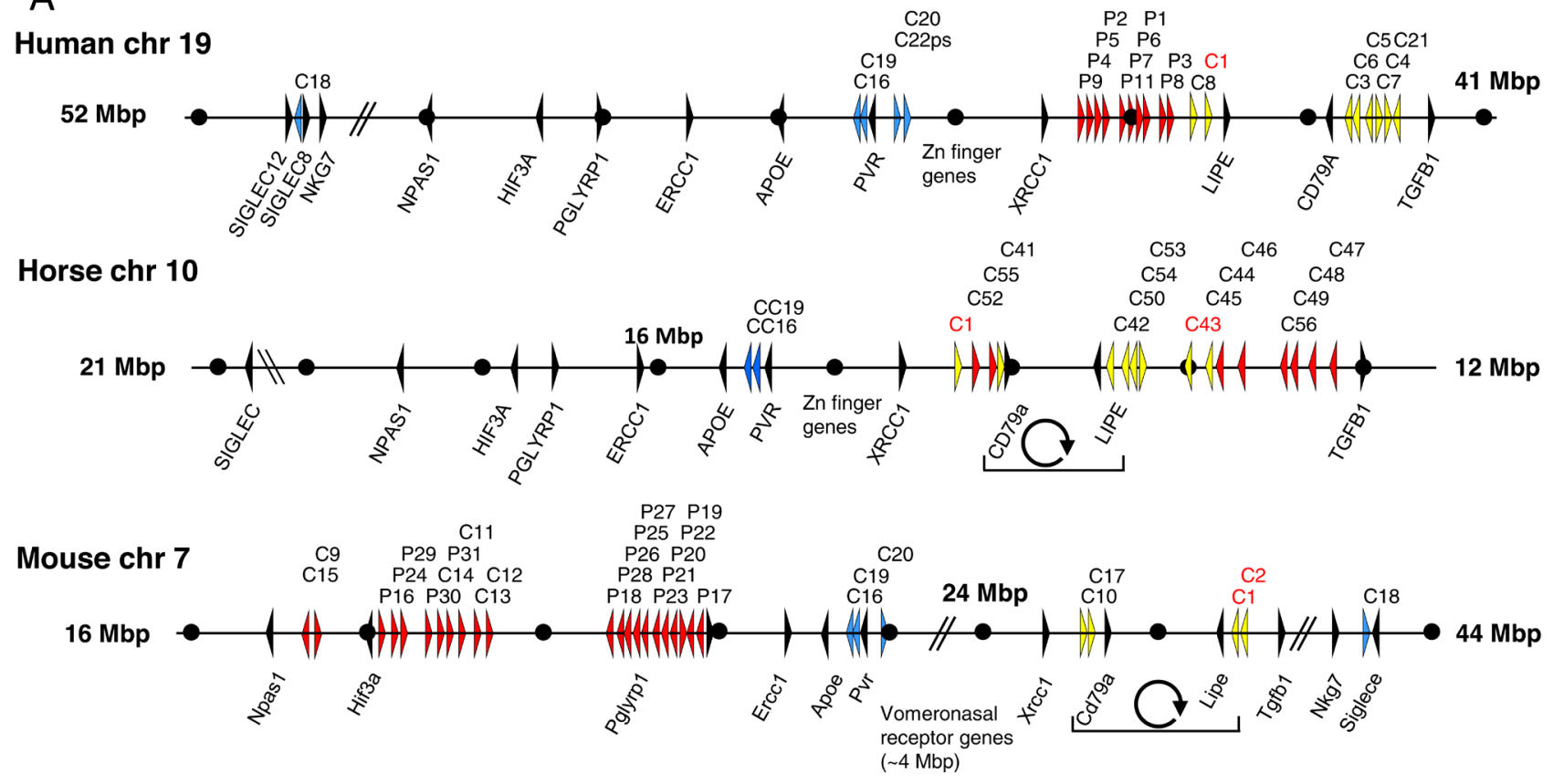

B

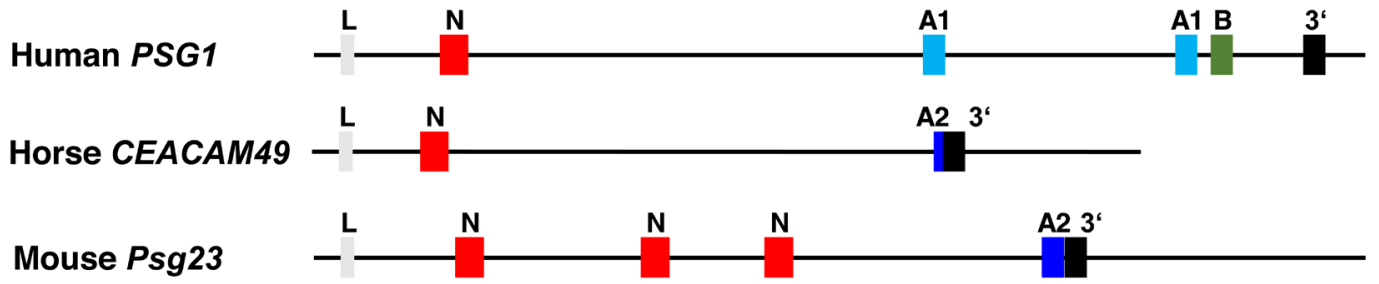

Figure 5 Syntenic relationship of the CEACAMIPSG gene loci and exon composition of PSG genes in the horse, mouse, and human genomes suggest independent evolution of PSG genes in the horse. (A) The syntenic chromosome regions from human, horse and mouse are shown. Arrowheads represent genes with their transcriptional orientation. CEACAM genes are indicated in yellow and PSG genes in red, the orthologous CEACAM genes in blue and marker genes in black. Names of CEACAM1-like genes with ITIM/ITSM motif-encoding exons are shown in red. Clockwise oriented arrows symbolize inversion events of the regions between the two CEACAM1-like gene clusters. Inversions relative to the human gene order are shown. Nucleotide numbering of the chromosomes starts at the telomere of the short arms. The scale indicated by dots is $1 \mathrm{Mbp}$ unless interrupted by slanted lines. C, CEACAM/Ceacam; chr, chromosome; P, PSG/Psg. (B) Exon arrangement of PSG genes in humans, horses and mice. The exon types are indicated by differently colored boxes. Leader sequences $(\mathrm{L})$ are shown as gray, IgV-like domain exons as red $(\mathrm{N})$, IgC-like domain exons as light blue (A1 type), green (B type) and blue (A2 type) and 3'-sequences as black boxes.

necessary strong selective pressure to drive convergent evolution of PSGs in different mammalian linages.

Despite all the functional similarities of PSGs in different mammalian species, there are fundamental differences between the hemochorial placenta of humans and mice and the placenta of equids. In the horse, the vast majority of the placenta is of the epitheliochorial type (Allen et al. 1973). Only a minority of trophoblast cells invade the endometrium, where they form the endometrial cups (Allen et al. 1973). These structures are unique in equids and have not been found in other mammals (Antczak et al. 2013). Although these structures are surrounded by a large amount of lymph vessels, which allow the transport of hormones, cytokines and other secreted products of the EC cells to the circulation, these trophoblast cells are not in direct contact with the blood of the mare (Antczak et al. 2013). Nevertheless, invading chorionic girdle cells and EC cells secrete PSGs in the horse. In humans, the main source of PSGs is the syncytiotrophoblast, which is in direct contact with maternal blood. Thus, tissue penetration of PSGs in the horse placenta is much more challenging than for PSGs in the hemochorial placentas of the human and mouse. We, therefore, hypothesize that the nanoscale size (single IgV-like domain, which is one-tenth of the size of an $\lg \mathrm{G}$ antibody) of equine PSGs are an adaptation to their high requirements on tissue penetration (Aleksic et al. 2016).

The horse is the only mammalian species in which a strong immune response to paternal antigens can be regularly detected. This may be because invading trophoblast cells (chorionic girdle cells) express, at least temporarily, conventional MHC class I molecules on 
the cell surface, therefore presenting semi-allogeneic antigens to the immune system of the mare. Consequently, maternal lymphoid cells migrate to EC and build a kind of demarcating zone at the edge of the EC. Nevertheless due to unknown mechanisms, the lymphoid cells do not attack and destroy EC until around day 130 of pregnancy (Antczak et al. 2013). More recently, it was described that the lymphocyte infiltrate consists of predominantly $\mathrm{T}$ cells and that a high frequency of these cells express FoxP3, a marker of Tregs (Noronha \& Antczak 2010). Differentiation and maturation of Tregs is favored by TGFB and we determined that the two PSGs examined, PSG1 and PSG9, induced the generation of Tregs following activation of naïve T cells with anti-CD3 and anti-CD28 antibodies and addition of IL-2 in a TGFB dependent manner (Jones et al. 2019). Tregs are essential for suppressing destructive alloantigenic immunity during pregnancy in mice (Zenclussen 2006, Tsuda et al. 2019). In humans, Tregs were decreased in peripheral blood and decidua in women with miscarriages and an impaired suppressive capability of Tregs in recurrent miscarriage cases was also observed (Tsuda et al. 2019). Furthermore, early pregnancy loss correlated with a low level of naturally occurring Tregs in mares (Aurich et al. 2014). Taken together, these observations indicate that equine PSGs, like human and murine PSGs, may play a role in the generation of Tregs via activation of TGFB, and therefore are important to maintain immune tolerance during pregnancy.

\section{Declaration of interest}

Tom Moore is on the editorial board of Reproduction. Tom Moore was not involved in the review or editorial process for this paper, on which he is listed as an author. The other authors have nothing to disclose.

\section{Funding}

This work was supported by the DFG (HE 6249/4-1 to R K), the National Institutes of Health (R21Al120918 to G D) and the Collaborative Health Initiative Research Program at USUHS (401738 to G D). A B was supported by the Intramural Research Program number NS002945 of the NINDS, NIH, Bethesda, MD, to Kenton J Swartz.

\section{Author contribution statement}

$\mathrm{R} K$ conceived the study, did biocomputing and manuscript writing. A B performed experiments and graphed the data. D $B$ generated and purified recombinant proteins. J W performed experiments and graphed the data. J M W performed experiments. T M supervised protein production and aided in the manuscript writing. G D designed experiments and aided in the manuscript writing. All authors participated in the design of the study and read and approved the final version.

\section{Acknowledgements}

The authors would like to thank Dr Esther Klaile (University Hospital Jena, Germany) and Dr Bernhard B Singer (University Hospital Essen, Germany) for providing CEACAM plasmids.

\section{References}

Abe M, Harpel JG, Metz CN, Nunes I, Loskutoff DJ \& Rifkin DB 1994 An assay for transforming growth factor-beta using cells transfected with a plasminogen activator inhibitor-1 promoter-luciferase construct. Analytical Biochemistry 216 276-284. (https://doi.org/10.1006/abio.1994.1042)

Aleksic D, Blaschke L, Mißbach S, Hänske J, Weiß W, Handler J, Zimmermann W, Cabrera-Sharp V, Read JE, de Mestre AM et al. 2016 Convergent evolution of pregnancy-specific glycoproteins in human and horse. Reproduction 152 171-184. (https://doi.org/10.1530/REP-16-0236)

Allen WR, Hamilton DW \& Moor RM 1973 The origin of equine endometrial cups. II. Invasion of the endometrium by trophoblast. Anatomical Record 177 485-501. (https://doi.org/10.1002/ar.1091770403)

Annes JP, Munger JS \& Rifkin DB 2003 Making sense of latent TGFbeta activation. Journal of Cell Science 116 217-224. (https://doi.org/10.1242/ jcs.00229)

Antczak DF, de Mestre AM, Wilsher S \& Allen WR 2013 The equine endometrial cup reaction: a fetomaternal signal of significance. Annual Review of Animal Biosciences 1 419-442. (https://doi.org/10.1146/ annurev-animal-031412-103703)

Aurich C, Weber J, Nagel C, Merkl M, Jude R, Wostmann S, Ollech D, Baron U, Olek S \& Jansen T 2014 Low levels of naturally occurring regulatory $\mathrm{T}$ lymphocytes in blood of mares with early pregnancy loss. Reproduction, Fertility, and Development 26 827-833. (https://doi. org/10.1071/RD13012)

Ballesteros A, Mentink-Kane MM, Warren J, Kaplan GG \& Dveksler GS 2015 Induction and activation of latent transforming growth factorbeta1 are carried out by two distinct domains of pregnancy-specific glycoprotein 1 (PSG1). Journal of Biological Chemistry 290 4422-4431. (https://doi.org/10.1074/jbc.M114.597518)

Beauchemin N \& Arabzadeh A 2013 Carcinoembryonic antigenrelated cell adhesion molecules (CEACAMs) in cancer progression and metastasis. Cancer Metastasis Reviews 32 643-671. (https://doi. org/10.1007/s10555-013-9444-6)

Blois SM, Tirado-Gonzalez I, Wu J, Barrientos G, Johnson B, Warren J, Freitag N, Klapp BF, Irmak S, Ergun S et al. 2012 Early expression of pregnancy-specific glycoprotein 22 (PSG22) by trophoblast cells modulates angiogenesis in mice. Biology of Reproduction 86191. (https://doi.org/10.1095/biolreprod.111.098251)

Blois SM, Sulkowski G, Tirado-Gonzalez I, Warren J, Freitag N, Klapp BF, Rifkin D, Fuss I, Strober W \& Dveksler GS 2014 Pregnancy-specific glycoprotein 1 (PSG1) activates TGF-beta and prevents dextran sodium sulfate (DSS)-induced colitis in mice. Mucosal Immunology 7 348-358. (https://doi.org/10.1038/mi.2013.53)

Bonsor DA, Gunther S, Beadenkopf R, Beckett D \& Sundberg EJ 2015 Diverse oligomeric states of CEACAM IgV domains. PNAS 112 13561-13566. (https://doi.org/10.1073/pnas.1509511112)

Chan WY, Tease LA, Bates Jr JM, Borjigin J \& Shupert WL 1988 Pregnancyspecific beta 1 glycoprotein in rat: tissue distribution of the mRNA and identification of testicular cDNA clones. Human Reproduction 3 687-692. (https://doi.org/10.1093/oxfordjournals.humrep.a136767)

de Mestre A, Noronha L, Wagner B \&Antczak DF 2010 Split immunological tolerance to trophoblast. International Journal of Developmental Biology 54 445-455. (https://doi.org/10.1387/ijdb.082795ad)

Derynck R \& Budi EH 2019 Specificity, versatility, and control of TGFbeta family signaling. Science Signaling 12 eaav5183. (https://doi. org/10.1126/scisignal.aav5183)

Falcon CR, Martinez FF, Carranza F, Cervi L \& Motran CC 2014 In vivo expression of recombinant pregnancy-specific glycoprotein 1a inhibits the symptoms of collagen-induced arthritis. American Journal of Reproductive Immunology 72 527-533. (https://doi.org/10.1111/ aji.12307)

Hadaschik EN \& Enk AH 2015 TGF-beta1-induced regulatory T cells. Human Immunology 76 561-564. (https://doi.org/10.1016/j. humimm.2015.06.015) 
Jones RL, Stoikos C, Findlay JK \& Salamonsen LA 2006 TGF-beta superfamily expression and actions in the endometrium and placenta. Reproduction 132 217-232. (https://doi.org/10.1530/rep.1.01076)

Jones K, Bryant S, Luo J, Kiesler P, Koontz S, Warren J, Malech H, Kang E \& Dveksler G 2019 Recombinant pregnancy-specific glycoprotein 1 has a protective role in a murine model of acute graft-versus-host disease. Biology of Blood and Marrow Transplantation 25 193-203. (https://doi. org/10.1016/j.bbmt.2018.09.022)

Kammerer R \& Zimmermann W 2010 Coevolution of activating and inhibitory receptors within mammalian carcinoembryonic antigen families. BMC Biology 8 12. (https://doi.org/10.1186/1741-7007-8-12)

Kammerer R, Mansfeld M, Hanske J, Missbach S, He X, Kollner B, Mouchantat S \& Zimmermann W 2017 Recent expansion and adaptive evolution of the carcinoembryonic antigen family in bats of the Yangochiroptera subgroup. BMC Genomics 18 717. (https://doi. org/10.1186/s12864-017-4106-7)

Kromer B, Finkenzeller D, Wessels J, Dveksler G, Thompson J \& Zimmermann W 1996 Coordinate expression of splice variants of the murine pregnancy-specific glycoprotein (PSG) gene family during placental development. European Journal of Biochemistry 242 280-287. (https://doi.org/10.1111/j.1432-1033.1996.0280r.x)

Kumar S, Stecher G, Li M, Knyaz C \& Tamura K 2018 MEGA X: molecular evolutionary genetics analysis across computing platforms. Molecular Biology and Evolution 35 1547-1549. (https://doi.org/10.1093/molbev/ msy096)

Laayouni $\mathbf{H}$, Oosting $M$, Luisi P, loana $M$, Alonso S, Ricano-Ponce I, Trynka G, Zhernakova A, Plantinga TS, Cheng SC et al. 2014 Convergent evolution in European and Rroma populations reveals pressure exerted by plague on toll-like receptors. PNAS 111 2668-2673. (https://doi. org/10.1073/pnas.1317723111)

Lea RG, Stewart F, Allen WR, Ohno I \& Clark DA 1995 Accumulation of chromotrope $2 \mathrm{R}$ positive cells in equine endometrium during early pregnancy and expression of transforming growth factor-beta 2 (TGFbeta 2). Journal of Reproduction and Fertility 103 339-347. (https://doi. org/10.1530/jrf.0.1030339)

Lennard SN, Stewart F \& Allen WR 1995 Transforming growth factor beta 1 expression in the endometrium of the mare during placentation. Molecular Reproduction and Development 42 131-140. (https://doi. org/10.1002/mrd.1080420202)

Lienart S, Merceron R, Vanderaa C, Lambert F, Colau D, Stockis J, van der Woning B, De Haard H, Saunders M, Coulie PG et al. 2018 Structural basis of latent TGF-beta1 presentation and activation by GARP on human regulatory T cells. Science 362 952-956. (https://doi.org/10.1126/ science.aau2909)

Lisboa FA, Warren J, Sulkowski G, Aparicio M, David G, Zudaire E \& Dveksler GS 2011 Pregnancy-specific glycoprotein 1 induces endothelial tubulogenesis through interaction with cell surface proteoglycans. Journal of Biological Chemistry 286 7577-7586. (https:// doi.org/10.1074/jbc.M110.161810)

Martinez FF, Knubel CP, Sanchez MC, Cervi L \& Motran CC 2012 Pregnancy-specific glycoprotein $1 \mathrm{a}$ activates dendritic cells to provide signals for Th17-, Th2-, and Treg-cell polarization. European Journal of Immunology 42 1573-1584. (https://doi.org/10.1002/eji.201142140)

Missbach S, Aleksic D, Blaschke L, Hassemer T, Lee KJ, Mansfeld M, Hanske J, Handler J \& Kammerer R 2018 Alternative splicing after gene duplication drives CEACAM1-paralog diversification in the horse. BMC Evolutionary Biology 18 32. (https://doi.org/10.1186/s12862-018-1145-x)

Moore T \& Dveksler GS 2014 Pregnancy-specific glycoproteins: complex gene families regulating maternal-fetal interactions. International Journal of Developmental Biology 58 273-280. (https://doi.org/10.1387/ ijdb.130329gd)

Morikawa M, Derynck R \& Miyazono K 2016 TGF-beta and the TGF-beta family: context-dependent roles in cell and tissue physiology. Cold Spring Harbor Perspectives in Biology 8 a021873. (https://doi.org/10.1101/ cshperspect.a021873)

Motran CC, Diaz FL, Montes CL, Bocco JL \& Gruppi A 2003 In vivo expression of recombinant pregnancy-specific glycoprotein 1a induces alternative activation of monocytes and enhances Th2-type immune response. European Journal of Immunology 33 3007-3016. (https://doi. org/10.1002/eji.200323993)
Naghibalhossaini F \& Ebadi P 2006 Evidence for CEA release from human colon cancer cells by an endogenous GPI-PLD enzyme. Cancer Letters 234 158-167. (https://doi.org/10.1016/j.canlet.2005.03.028)

Noronha LE \& Antczak DF 2010 Maternal immune responses to trophoblast: the contribution of the horse to pregnancy immunology. American Journal of Reproductive Immunology 64 231-244. (https://doi. org/10.1111/j.1600-0897.2010.00895.x)

Rattila S, Dunk CEE, Im M, Grichenko O, Zhou Y, Yanez-Mo M, Blois SM, Yamada KM, Erez O, Gomez-Lopez N et al. 2019 Interaction of pregnancy-specific glycoprotein 1 with integrin alpha5beta1 is a modulator of extravillous trophoblast functions. Cells 81369.

Robertson IB, Horiguchi M, Zilberberg L, Dabovic B, Hadjiolova K \& Rifkin DB 2015 Latent TGF-beta-binding proteins. Matrix Biology 47 44-53. (https://doi.org/10.1016/j.matbio.2015.05.005)

Robertson IB \& Rifkin DB 2016 Regulation of the bioavailability of TGFbeta and TGF-beta-related proteins. Cold Spring Harbor Perspectives in Biology 8 a021907. (https://doi.org/10.1101/cshperspect.a021907)

Rudert F, Saunders AM, Rebstock S, Thompson JA \& Zimmermann W 1992 Characterization of murine carcinoembryonic antigen gene family members. Mammalian Genome 3 262-273. (https://doi.org/10.1007/ BF00292154)

Shanley DK, Kiely PA, Golla K, Allen S, Martin K, O'Riordan RT, Ball M, Aplin JD, Singer BB, Caplice N et al. 2013 Pregnancy-specific glycoproteins bind integrin alphallbbeta 3 and inhibit the platelet-fibrinogen interaction. PLOS ONE 8 e57491. (https://doi.org/10.1371/journal.pone.0057491)

Shi M, Zhu J, Wang R, Chen X, Mi L, Walz T \& Springer TA 2011 Latent TGF-beta structure and activation. Nature 474 343-349. (https://doi. org/10.1038/nature10152)

Singer BB, Opp L, Heinrich A, Schreiber F, Binding-Liermann R, BerrocalAlmanza LC, Heyl KA, Muller MM, Weimann A, Zweigner J et al. 2014 Soluble CEACAM8 interacts with CEACAM1 inhibiting TLR2-triggered immune responses. PLOS ONE 9 e94106. (https://doi.org/10.1371/ journal.pone.0094106)

Stockis J, Colau D, Coulie PG \& Lucas S 2009 Membrane protein GARP is a receptor for latent TGF-beta on the surface of activated human Treg. European Journal of Immunology 39 3315-3322. (https://doi. org/10.1002/eji.200939684)

Streydio C, Lacka K, Swillens S \& Vassart G 1988 The human pregnancyspecific beta 1-glycoprotein (PS beta G) and the carcinoembryonic antigen (CEA)-related proteins are members of the same multigene family. Biochemical and Biophysical Research Communications 154 130-137. (https://doi.org/10.1016/0006-291x(88)90660-2)

Tsuda S, Nakashima A, Shima T \& Saito S 2019 New paradigm in the role of regulatory T cells During pregnancy. Frontiers in Immunology 10573. (https://doi.org/10.3389/fimmu.2019.00573)

Warren J, Im M, Ballesteros A, Ha C, Moore T, Lambert F, Lucas S, Hinz B \& Dveksler G 2018 Activation of latent transforming growth factorbeta1, a conserved function for pregnancy-specific beta 1-glycoproteins. Molecular Human Reproduction 24 602-612. (https://doi.org/10.1093/ molehr/gay044)

Watanabe S \& Chou JY 1988 Human pregnancy-specific beta 1-glycoprotein: a new member of the carcinoembryonic antigen gene family. Biochemical and Biophysical Research Communications 152 762-768. (https://doi.org/10.1016/s0006-291x(88)80103-7)

Zenclussen AC 2006 Regulatory T cells in pregnancy. Springer Seminars in Immunopathology 28 31-39. (https://doi.org/10.1007/s00281-0060023-6)

Zhou GQ \& Hammarstrom S 2001 Pregnancy-specific glycoprotein (PSG) in baboon (Papio hamadryas): family size, domain structure, and prediction of a functional region in primate PSGs. Biology of Reproduction 64 90-99. (https://doi.org/10.1095/biolreprod64.1.90)

Received 21 May 2020

First decision 24 June 2020

Revised manuscript received 10 July 2020

Accepted 7 August 2020 